\title{
The Passion behind Serenity
}

\author{
Xiaoyan Yang \\ School of Art and Garment \\ Tianjin Polytechnic University \\ Tianjin 300384, China \\ E-mail: yang831006@126.com
}

\begin{abstract}
It forms certain sense of beauty in the asymmetry in symmetry, among the shape bodies of oil paintings, between body shape and image as well as the both symmetrical-and-asymmetrical-like objects in the natural world. The structure lines in drawing creation cross each other, are connected with each other and collide with each other, which forms a tense atmosphere, transforms into an instant balance and a kind of comparatively static beauty, and strongly expresses the visual effects and the mood it intends to express.
\end{abstract}

Keywords: Asymmetry, Collision, Balance, Static

Someone says that inspiration comes from suddenness and is beyond human beings' expectation. Perhaps a coincidence, a casual glance, and a moment when enjoying a photo of Tianshan snow will bring the germinating of inspiration. For example, when we stand still in creation, the chance of seeing a landscape painting will bring you new ideas. Simple composition and easy brushwork help us ignite the passion for painting.

From the perspective of creation materials, human being's appreciation on the asymmetry beauty of the natural works has a great potential. It includes the mutual reflection between water and mountain, the mutual reflection between mountain and their images reflected in the water and the mutual relationship between the sky and coast. The world of "asymmetry" is dulcet and is the beauty of serenity.

Most sceneries in the nature have certain symmetry so as to keep balance. However, the colorfulness of the world usually lies in its not-so-complete symmetry. A left-and-right symmetrical mountain and water painting could give people the feelings of beauty. However, if we combine half of the painting with its image and form an absolute symmetrical mountain and water painting, the effect will be totally different. Such absolute symmetrical painting is dull and has no similarity with the natural scenery that is full of energy and has no sense of beauty at all. If we break the symmetry or balance, even the slightest breaking, it will bring the beautiful effect out of expectation. Imagine that there is a painting that describes mountain and water scenery. The mountain and water are symmetrical in the entire painting. You will feel the disharmony of this painting, which is caused by the over symmetry of the painting. Although to break the symmetry could generate power, in the gale and swell whose surface is turbulent, even a cluster of grass or a boat, who are struggling for balance, will bring forceful reactions. The symmetrical beauty of the nature once makes many people admire. The principle of symmetry and asymmetry in the nature that we discover is similar to the aesthetic principle of symmetry and asymmetry that artists discover and explore.

The asymmetry beauty in painting is just one aspect. The creation of works needs painting skills to represent so as to increase the agile beauty. The skill and game nature of the creation of oil painting attract people to learn and explore. For example, in creation, we could completely break the trueness and small strokes and could boldly try to apply big strokes concept and to try something new.

The skills that combine the skills of abstractive painting and impressionist painting provide us with the possibility to fully express our feelings in painting creation. We could use various skills to form the static beauty after collision. For example, we could use color module to express entity and could use the squeezing of color modules to express the relationship between various bodies. The modern art admires concepts not experiencing. It pays less attention to the techniques of creation. As a matter of fact, not only oil painting needs skills, concept art, behavior art and installation are also need skills. They are just of different styles.

The squeezing of color modules could form a static beauty after collision. Therefore, light sense could also generate the 
sense of beauty. Any object will generate light and shade changes what put under light. For example, the light and shade effect generated when a ball is placed under light will give people with a visual impact. For another example, the "focusing and dispersing" concept in 3 Dimension refers to that when light reaches object surfaces, it will reflect and generate the glisten like water wave. The entire light refers to the whole balance and the focusing and dispersing effect means the un-balance in balance, which could form a collision beauty after collision in common and change. Boudelaire said that art is composed of objective elements, common and change, eternal and instant, absolute and incidental. He believed that a great art should discover eternal elements in instant ones, discover absolute elements in incidental ones, and see common elements in changing elements.

In the formation and representation of painting works, there is passion. The crossing of curved lines and beelines forms a tense atmosphere. Such status suddenly become still in an instant balance or movement. This is an instant concept, a beauty that comes when mountain waters suddenly stop. It is believed that a good start means half success of a career. It is so in painting.

All lights of beauty come from our heart. Without heart reflection, there is no so called beauty. Therefore, the natural scenery is the field of heart and soul. Especially for the conception paintings in Chinese paintings, they lie between "similarity and non-similarity" and are called "the unification of heaven and human beings", the cultural spirit of China. They instinctively combine representation with reappearance and subjectivity and objectivity. Such instinct sense and the comprehensive application fundamentally distinguish Chinese thinking patterns with the linear logistic method and aesthetic modes of western culture. What is more, for aesthetic "image", it includes sense, thoughts, and spirit and at the same time, it is the reflection of culture, personality ambit and taste style. Hence, from "image", there come three cultural concepts," artistic concept", "ambit" and "style", which are related to works but could be not specified. They refer to the humanism culture and personality connotation of the artistic main bodies reflected by the artistic works. From the perspective of oil painting, image oil paintings could best show the poem cultural oil painting. In the creation concepts, image oil painting pursues "go with the objects" and "follow the direction of hearts", which means that for one aspect, it should focus on the objects and heart should yield to the objects. For another aspect, it requires that to use heart to direct objects. The works might not just reflect the true objects but to certain degree, it keeps the certain relationship with real images.

No matter what methods, styles and patterns are adopted, for the art that is realistic and reflect the true feelings of human beings, there is a broad road. The more successful point of impressionism painters is that they bring people back to the nature. They advocate sketch, trueness, and return to the nature. Hence, people now yearn for "going to the nature". Due to the sub-consciousness, some painters want to change the trueness of this world. Of course, the trueness here does not refer to the physical property of objects and is a new ideal and transformed entity. For example, the sky and water are originally entities. However, due to the need of creation, they could be abstractive. What replaces them could be the pure color. It looks like mountain but is not mountain. It looks like water but is not water. It looks like cloud and is not cloud. Even, you could reverse the painting to appreciate it. Hence, you could feel the degree of the complanation and subjectivity of the works. The creation of oil paintings could adopt "color face" to talk. The height wave of line face in the painting could be squeezed out through "color face". In oil painting works, the main body structure formed due to the mutual squeezing of color module makes the shape of objects only leave the edge of color module. However, the structure lines hided in the painting cross each other, compare with each other, are connected with each other so that a type of comparative static beauty is formed. By this way, the true objects seem like transient and mysterious. What is more, it pursues the pureness and simplicity of color image as well as the pureness and romantics of color so that those non-realistic objects are completely integrated in to the cloud stream like color change. This is the artistic conception what we pursue in painting creation.

Usually, in an excellent works, there is a type of turbulence hidden behind serenity. At the first glance, it will give people with warm, placid, gentle and amiable memory and feelings. Its colors change slightly, is pure but not single and deserves your memory. Its shapes have a type of comparative stillness in collision, which has a force that controls us. As a matter of fact, it is beautiful poem. In the painting, you could feel the beauty of movements and stillness. When the movements and stillness collide with each other, it forms a type of balance and a comparative serenity.

All objects own the beauty of asymmetry and at the same time, they all have objective aspects. No matter it is in the vast universe or in the daedal natural world, the subjective concepts of painters collide with each other and form a beauty of balance and serenity. Behind the beauty of serenity, it is the passion for creation.

\section{References}

Baudelaire. Baudelaire Aesthetic Papers. Translated by Guo, Hongan in 1987. Beijing, People's Literature Press.

Long, Rui. (2002). Art Observation. Beijing: Art Observation Press.

Chen, Chiyu. (1996). Art Comments. Wuhan: Huazhong Normal University Press.

Xiao, Mo. (2004). Aesthetics and Art Appreciation. Beijing: Higher Education Press

Yang, Gaixue. (2004). Modern Visual Media Art. Beijing: Higher Education Press. 